\title{
The influence of asphalt ageing on induction healing effect on porous asphalt concrete
}

\author{
Shi $\mathrm{Xu}^{1, *}$, Xueyan Liu ${ }^{1}$, Amir Tabaković ${ }^{1,2,3}$, Erik Schlangen ${ }^{1}$ \\ ${ }^{1}$ Civil Engineering and Geosciences, Delft University of Technology, The Netherlands \\ 2 Research Enterprise and Innovation, Technological University Dublin, Dublin, Ireland \\ ${ }^{3}$ School of Civil Engineering, University College Dublin, Dublin, Ireland
}

Received: 12 December 2018 / Accepted: 15 February 2019 / Published online: 18 February 2019

(c) The Author(s) 2019. This article is published with open access and licensed under a Creative Commons Attribution 4.0 International License.

\begin{abstract}
Induction healing is a proven technology which is able to improve the self-healing capacity of asphalt concrete. Healing is achieved via electromagnetic current produced by passing induction machine, where steel asphalt constituents heat up which in turn soften the bitumen in the asphalt layer, allowing it to flow and close cracks, repairing the damage. This paper reports on the study which investigated the influence of ageing on the healing capacity of Porous Asphalt (PA) concrete. Porous Asphalt concrete mix was prepared first, then subjected to an accelerated (laboratory) ageing process using a ventilated oven. In order to further evaluate the induction healing efficiency of asphalt concrete, Semi-circular bending (SCB) and healing cycles were performed on asphalt concrete specimens. The results show that with an increase of the ageing level of porous asphalt concrete, the healing efficiency of the asphalt decreases.
\end{abstract}

Keywords: Induction heating; Asphalt; Semi-circular bending; Ageing; Self-healing

\section{Introduction}

Asphalt concrete when subjected to loading and environmental (ultraviolet radiation, oxidation and moisture damage) conditions leads to deterioration to its physical and mechanical properties, such as ageing [1-3]. On a molecular level, the ageing of asphalt involves synergy of several effects, such as: volatilisation, oxidation and steric hardening [3]. The volatilisation and oxidation result from the change in molecular structure, while steric hardening is a result of a molecular rearrangement [3]. In mechanical way, ageing of asphalt increases the stiffness and bitumen viscosity and eventually leads to ravelling and cracking in asphalt concrete [4-6]. The ageing effect of an asphalt concrete is influenced by time, temperature and asphalt concrete layer depth $[7,8]$. Therefore, a higher temperature or longer exposure period increase the asphalt ageing level, which changes the rheology of asphalt binder and reduces its ability to flow.

The asphalt concrete possesses an intrinsic healing capacity which allows it to heal the damage itself during the rest period [9]. Researchers have found that the self-healing ability of asphalt concrete is largely affected by the ambient temperature, which a higher temperature not only increases the self-healing rate but also shortens the healing period for a full recovery [9-11]. This healing effect can be illustrated by the time-temperature superposition of asphalt material in the following formulation [10]:

$$
\begin{gathered}
H(t, T)=100 \times\left[1+\left(\frac{m}{t \times a_{T}}\right)^{\frac{\log 2}{n}}\right]^{-\frac{n}{\log 2}} \\
\log a_{T}(T)=\frac{\Delta E_{a}}{2.303 R}\left(\frac{1}{T}-\frac{1}{T_{0}}\right)
\end{gathered}
$$

where:

$$
\begin{array}{ll}
\mathrm{m}, \mathrm{n} & =\text { model parameters; } \\
a_{T} & =\text { time-temperature superposition shift factor; } \\
\Delta E_{a} & =\text { apparent activation energy, } \mathrm{J} / \mathrm{mol} ; \\
\mathrm{R} & =\text { universal gas constant, } 8.314 \mathrm{~J} /(\mathrm{mol} \cdot \mathrm{K}) .
\end{array}
$$

Because of lower ambient field temperature, this self-healing effect of asphalt concrete is usually limited.

In the past 10 years, the induction healing has been intensively investigated as a novel method to achieve crack healing and eventually prolong the service life of an asphalt concrete [12-17]. The induction healing has been demonstrated to have a significant crack healing effect in asphalt mastic, porous asphalt concrete, dense asphalt concrete and reclaimed asphalt pavement (RAP) [18-20]. Healing is initiated within the asphalt by sending an alternating current through the coil and generating an alternating electromagnetic field. When the conductive

* Corresponding author: Shi Xu, E-mail: s.xu-1@tudelft.nl, Tel.: +31(0)-15-27-85944 
asphalt specimen is placed beneath the coil, this electromagnetic field induces currents flowing along the conductive loops formed by steel fibres. The current causes steel fibres to heat up which heats the aged bitumen and softens it, allowing it to flow and close the cracks, and to repair the damage. This method can be repeated if damage returns [9]. The induction heating method has a significant advantage in healing efficiency over the other existing selfhealing technologies, such as: rejuvenator encapsulation, for asphalt concrete and it has already been applied in field trials $[9,21,22]$.

Up to now, most researches investigating induction healing effect in asphalt used testing samples prepared with virgin asphalt mixes, which means the intrinsic healing capacity of the tested mixture is relatively high and can be easily stimulated via induction heating $[12-16,18]$. However, the optimum time to apply induction healing in asphalt concrete is at the crack (microcracks) initiation stage [9]. At this time, the asphalt concrete experiences aging, and its intrinsic healing capacity is reduced, this also could lead to reduced efficiency of extrinsic (induction) healing system [10, 23, 24].

Gómez-Meijide et al [19] considered the ageing level of asphalt binder as an induction healing influencing factor by testing asphalt concrete prepared with artificially aged RAP. Gómez-Meijide et al concluded that the ageing effect reduces the effectiveness and energy efficiency of induction healing treatments [19]. However, they indicated that the ageing level of asphalt mixture could affect the compaction rate of asphalt concrete [19], so that the inhomogeneous compaction rates of the tested asphalt concrete samples could not fully show the ageing effect on induction healing treatments.

The key objective of this study was investigation of the influence of ageing on asphalt concrete induction healing efficiency. Since porous asphalt is prone to ageing due its open structure, and shows better induction healing potential [19], it is selected as the mixture type in this study. At first, the porous asphalt concrete slabs were subjected to an accelerated laboratory ageing in order to simulate the in-situ asphalt ageing. Nano CT scan was employed to visualize the distribution of steel fibres within an asphalt mix. Then, the induction healing efficiency of SCB specimens were investigated, and the results between aged and virgin specimens were compared. The results demonstrate that the effect of ageing could have a huge impact on induction healing, which not only reduces the possible healing cycles but also reduces the healing efficiency.

\section{Methods}

\subsection{Porous asphalt concrete}

In an effort to evaluate the effect of ageing on the healing efficiency of the asphalt induction healing, a PA asphalt mix was designed (Table 1). All aggregates (including sand) used in this paper were limestones or by-product of limestones. The 70/100 bitumen was used as asphalt binder and the filler type Wigro $60 \mathrm{k}$ was used. The steel fibres used in the asphalt mix has a diameter of $40 \mu \mathrm{m}$ and an average length around
$1.4 \mathrm{~mm}$. The advantage of using these short fibres is to allow these fibres homogeneously distributed in the porous asphalt concrete, thus avoid clogging.

In this study, two porous asphalt slabs were prepared in Rosmalen Heijmans infra BV with a designed void content of $20 \%$, following the standard PA 0/11 according to the Rationalisatie en Automatisering Grond-, Water- en Wegenbouw (RAW) 2005 which was used in effort to produce PA asphalt mix typically used as asphalt wearing courses used in The Netherlands $[25,26]$. The steel fibres were added with an extra volume in a ratio of 6:100 with the volume of bitumen. The calculated porous asphalt composition is shown in Table 1.

Table 1. Mix Composition of Porous Asphalt Concrete

\begin{tabular}{ll}
\hline Mix Constituent & \% Content in Mix \\
\hline $16 \mathrm{~mm}$ & 7.97 \\
$11.2 \mathrm{~mm}$ & 62.00 \\
$8 \mathrm{~mm}$ & 7.97 \\
$5.6 \mathrm{~mm}$ & 1.78 \\
$2 \mathrm{~mm}$ & 6.47 \\
$500 \mu \mathrm{m}$ & 2.06 \\
$180 \mu \mathrm{m}$ & 0.66 \\
$125 \mu \mathrm{m}$ & 0.66 \\
$63 \mu \mathrm{m}$ & 4.22 \\
\hline Bitumen(70/100) & 4.32 \\
Fibres(excl.) & 1.88 \\
\hline
\end{tabular}

Two asphalt slabs with size of $50 \times 50 \times 5 \mathrm{~cm}$ were prepared and one of them was aged in a ventilated oven following an artificial ageing method which was used by Tabaković [27] and $\mathrm{Xu}$ [28]: kept in a ventilated oven at $135^{\circ} \mathrm{C}$ for 4 hours and then a 4 days period under $85^{\circ} \mathrm{C}$, which simulates the field ageing of 15 years.

After preparation of the porous asphalt concrete, a cylinder sample with $33.5 \mathrm{~mm}$ in diameter and $48.5 \mathrm{~mm}$ in height was drilled and scanned with a Nano CT scanner at resolution of $20 \mu \mathrm{mto}$ investigate the distribution steel fibres in the PA mix.

\subsection{SCB tests}

In the laboratory, the SCB test is widely used to investigate cracking performance of asphalt concrete $[9,29,30]$. In this paper, the SCB samples were acquired by drilling and cutting from the asphalt slabs. As shown in Fig. 1a, the SCB specimens have the diameter of $100 \mathrm{~mm}$, the width of $50 \mathrm{~mm}$, the height of $50 \mathrm{~mm}$ and a notch size of $2 \times 10 \mathrm{~mm}$.

A universal testing machine (UTM) with a temperature control chamber was employed for the SCB tests. According to EN 12697-44:2010 [31], the SCB tests were performed under $0{ }^{\circ} \mathrm{C}$ with a displacement control of $5 \mathrm{~mm} / \mathrm{min}$ and the support span was $80 \mathrm{~mm}$ (Fig. 1b). For each testing condition, at least 6 samples were tested.

\subsection{Induction healing}

The induction healing were performed using an induction machine which has a capacity of $50 \mathrm{~kW}$ and at a frequency of $70 \mathrm{kHz}$. Fig. 2a shows the top view of the SCB specimen under induction healing. The distance between the induction coil and healing specimen was kept at $5 \mathrm{~mm}$ (Fig. 2b). In order to achieve an effective and homogeneous healing on all the 
specimens, the induction healing temperature was carefully controlled to reach $85{ }^{\circ} \mathrm{C}$ (Fig. 2C), which is regarded as the optimum temperature for induction healing. An infrared camera was used to monitor the whole induction healing process to control the induction healing process and avoid overheating on SCB specimens.

\subsection{Bending and healing cycles}

In order to evaluate the healing efficiency of the SCB specimens, a bending and healing programme was followed:

Step 1: The initial peak load of the specimen was measured by the first SCB test, followed by a 4 hours resting at $23{ }^{\circ} \mathrm{C}$ to allow the fractured specimen reached room temperature;

Step 2: The fractured two parts were gently put together and placed $5 \mathrm{~mm}$ below the induction coil. Then, the induction healing was conducted on both sides of the SCB specimen and the heated specimen was left at $23^{\circ} \mathrm{C}$ for 4 $\mathrm{h}$ to let the temperature cooling down.

Step 3: Subsequently, a resting period of 12 hours on the specimen was conditioned on a plain surface at $23^{\circ} \mathrm{C}$. In order to create a constant confinement to ensure the close of cracked surfaces, the specimen was carefully wrapped with tapes during the resting period;

Step 4: The SCB test to acquire the strength recovery of the specimen after healing. If the peak load was higher than $100 \mathrm{~N}$, step 2 to 4 would be repeated until the sample could not be healed anymore.
The induction healing effect was determined using induction healing index $(\mathrm{IH})$, which was calculated with the peak load measured from three SCB tests:

$$
\mathrm{IH}=\frac{\mathrm{C}_{\mathrm{x}}}{\mathrm{C}_{1}} \times 100 \%
$$

where:

$$
\begin{array}{ll}
\mathrm{IH} & =\text { the induction healing index (\%), } \\
\mathrm{C}_{1} & =\text { original peak load of the sample; } \\
\mathrm{C}_{\mathrm{x}} & =\text { tested peak load after } \mathrm{x} \text { cycles of healing. }
\end{array}
$$

\section{Results}

\subsection{CT scan image}

Fig. 3 shows one of the top view Nano CT scan images. In Fig. 3, shows all three different phases in the scanned porous asphalt, including steel fibres, aggregates and asphalt mastic. The steel fibres are presented in the brightest colour. That is due to the density of the steel fibres is $7.5 \mathrm{~kg} / \mathrm{m}^{3}$, which is significantly higher than aggregates and asphalt mastic $(<2.8$ $\mathrm{kg} / \mathrm{m}^{3}$ ). The small size of steel fibres allows uniformly distribution of the fibres within the asphalt mastic area, and with $6 \%$ of steel fibres, nearly every piece of mastic area contains steel fibres. As such, theoretically, as long as the induction energy can reach, all microcracks in asphalt mastic are able to be healed with induction healing. The figure further shows that by using steel fibres with a length of 1.4 $\mathrm{mm}$, the traditional clogging problem during mixing can be solved.

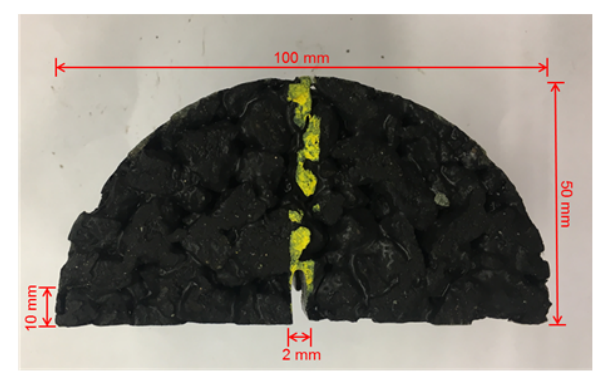

a

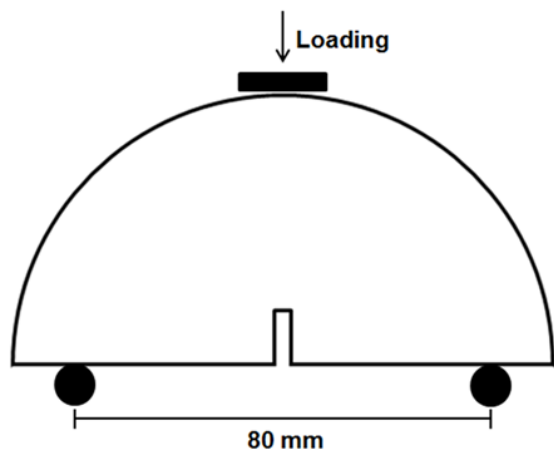

b

Figure 1. (a) SCB testing specimen; (b) loading on SCB samples.

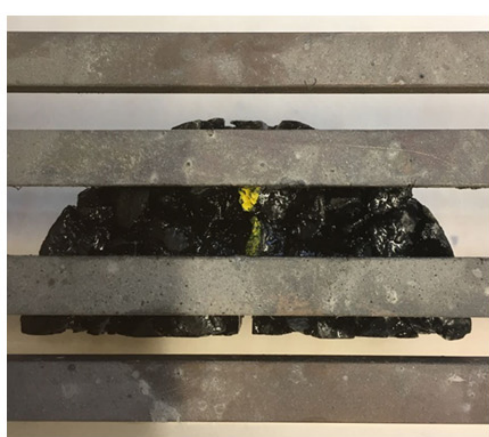

a

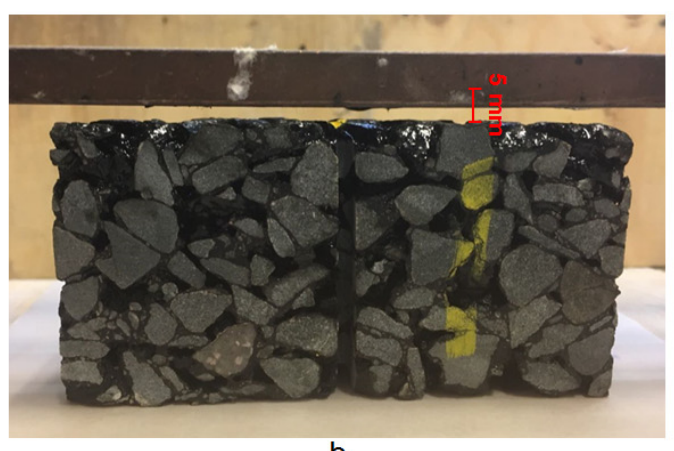

b

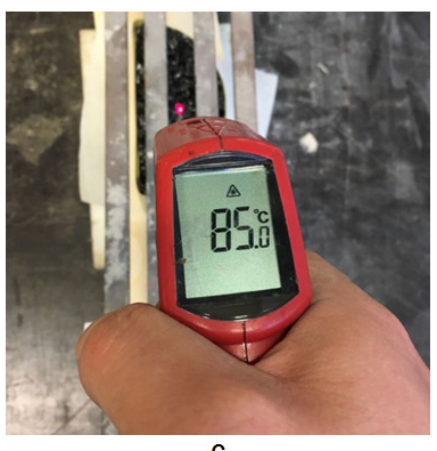

C

Figure 2. Tested SCB specimen under the induction coil: (a) top view; (b) front view and (c) surface temperature. 


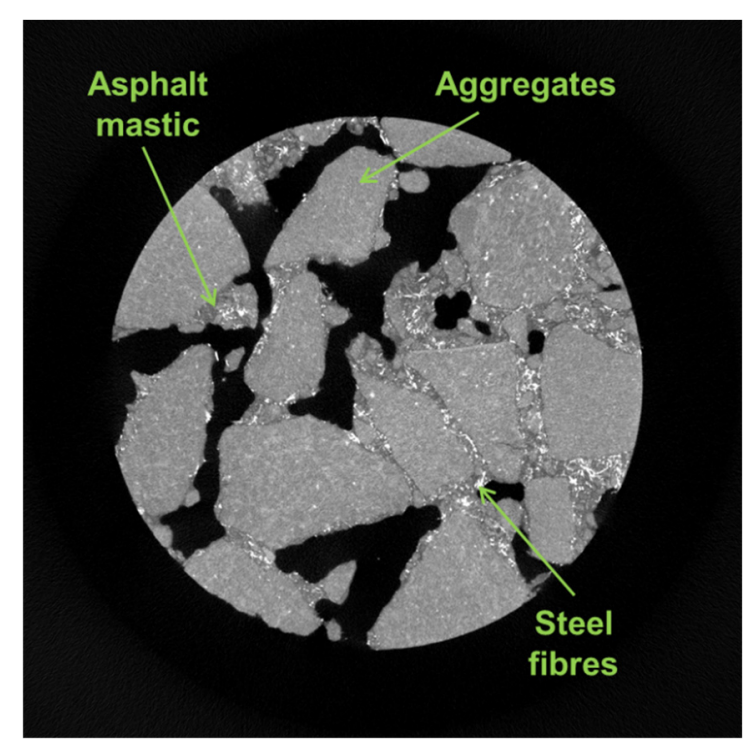

Figure 3. A top view image of CT scan on porous asphalt concrete with steel fibres.

\subsection{Induction healing process}

Fig. 4 shows the heat distribution throughout the full depth of the test specimen during the induction healing process. Fig. 4a illustrates an image of tests specimen prior the healing process at $23^{\circ} \mathrm{C}$. The specimen is shown in same colour as its surroundings under the infrared camera. The induction healing method used in this paper was applied in two steps:

I. When the induction healing starts, the alternating current through the coil generates an alternating electromagnetic field, leading to gradual temperature increase of the SCB specimen from the sample surface to the middle. This step lasted 90 seconds, and the infrared image shows that the temperature was the highest at the surface (Fig. 4d);

II. After one side heating was completed, the SCB specimen was turned over and the induction healing was applied on the other side for 60 seconds (Fig. 4c). Finally, after twosided healing, the infrared image showed the SCB specimen can achieve a uniform distribution of temperature about $85^{\circ} \mathrm{C}$, thus optimum healed.
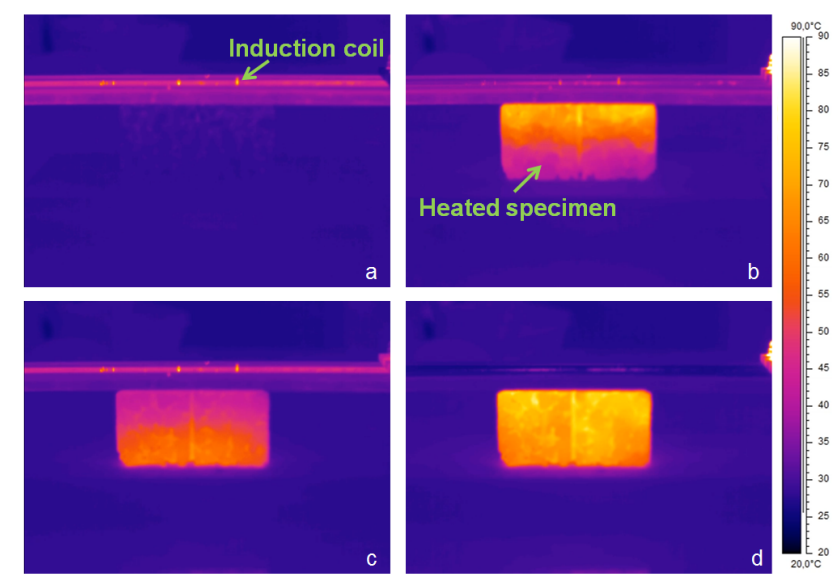

Figure 4. The healing process of SCB specimens: (a) heating start on one side; (b) one side heating complete; (c) Turn over and start heating on the other side and (d) the whole heating process complete.
In this way, the whole body of the heated sample reached $85^{\circ} \mathrm{C}$ (Fig. 4d), which is referred as the optimum temperature for induction healing. Thus, overheating and insufficient heating were able to be avoided. In practice, an uniform heating can be achieved by using a two-layer asphalt structure which has more fibres in the bottom-layer.

\subsection{SCB tests}

Fig. $5 a$ shows the peak load results from all SCB tests. In the beginning, the fresh PA specimens and the aged PA specimens have very similar initial peak load around $2.3 \mathrm{kN}$. As the number of bending and healing cycles increases, the regained peak load of all PA specimens decreases, which indicates a decreasing of fracture resistance. This general decreasing trend might because of the accelerated ageing of asphalt concrete by induction heating, which gradually deteriorates the self-healing capacity of PA mix and in turn decrease the induction healing efficiency (Fig. 5b).

After 6 healing cycles, the induction healing index of fresh asphalt specimens still reaches $73 \%$, while the aged asphalt specimens remain only $25 \%$, and some aged specimens cannot be healed with induction healing anymore. For aged PA specimens, the regained peak load is significantly lower than fresh ones. It might because, after the laboratory ageing, the self-healing capacity of the PA mix decreases, which means the high temperature produced from induction healing could not provide as much healing effect as the fresh PA mix.

a
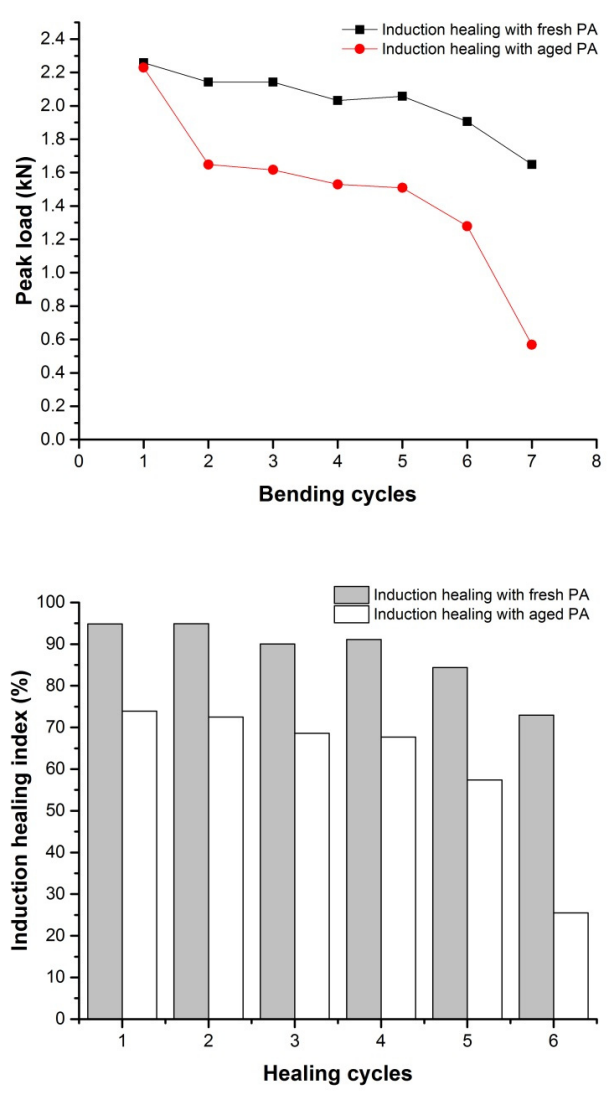

Figure 5. (a) Peak load of SCB specimens and (b) induction healing index of SCB specimens. 


\section{Conclusions}

This study investigated the effect of asphalt ageing on induction healing, the following conclusions are drawn:

- The CT scan image indicates that incorporation of induction healing technology using steel fibres with a length of $1.4 \mathrm{~mm}$ can achieve a homogeneous distribution of steel fibres in asphalt concrete. With $6 \%$ of steel fibres, as fibres are able to spread in every pieces of asphalt mastic, theoretically, cracks in the asphalt mastic can be healed via induction healing;

- The induction healing procedure adopted in this study, by applying induction healing on both sides of a specimen, can achieve a homogeneous temperature distribution on asphalt concrete, thus optimum healing;

- the PA mix induction healing index decreased with increasing number of healing cycles, which illustrate that the high temperature produced by induction healing can help to improve the self-healing capacity of an asphalt concrete, which in turn could also accelerate the asphalt ageing process;

- The higher ageing level in asphalt concrete results in a further reduction of induction healing effect. Ageing of an asphalt concrete not only decreases its induction healing efficiency but also reduces its possible healing cycles.

Finally, recognised the significance of asphalt ageing effect on induction healing, researchers could develop the induction healing technology in a more scientific way that predicts the induction healing behaviour in combination with the longterm service life of an asphalt concrete. On the other hand, considering the findings in this paper, it inspires a new way to prolong the service life of asphalt concrete with self-healing asphalt: incorporating induction healing system with capsule healing system, by this means, the crack healing and aged binder rejuvenation are combined, as such, a longer extended life span is expected.

\section{Acknowledgments}

The authors would like to thank Heijmans for the help in making all the porous asphalt specimens used in the research. Technical support from employees from the pavement engineering section and Microlab in TUDelft are also appreciated. Finally, the study was funded by China Scholarship Council (No. 201506950066).

\section{References}

[1] S. Wu, L. Pang, L. Mo, J. Qiu, G. Zhu, Y. Xiao, UV and thermal aging of pure bitumen-comparison between laboratory simulation and natural exposure aging, Road Mater Pavement Des (2008): 103-113. https://doi.org/10.1080/14680629.2008.9690161

[2] R. Micaelo, T. Al-Mansoori, A. Garcia, Study of the mechanical properties and self-healing ability of asphalt mixture containing calcium-alginate capsules, Constr Build Mater (2016) 123: 734-744. https://doi.org/10.1016/i.conbuildmat.2016.07.095

[3] I. Androjić, Ageing of hot mix asphalt, Građevinar (2016) 68: 477-483.

[4] J.-F. Su, J. Qiu, E. Schlangen, Y.-Y. Wang, Investigation the possibility of a new approach of using microcapsules containing waste cooking oil: In situ rejuvenation for aged bitumen, Constr Build Mater (2015) 74: 83-92. https://doi.org/10.1016/j.conbuildmat.2014.10.018
[5] G. Liu, T. Yang, J. Li, Y. Jia, Y. Zhao, J. Zhang, Effects of aging on rheological properties of asphalt materials and asphalt-filler interaction ability, Constr Build Mater (2018) 168: 501-511. https://doi.org/10.1016/j.conbuildmat.2018.02.171

[6] J.-F. Su, J. Qiu, E. Schlangen, Stability investigation of self-healing microcapsules containing rejuvenator for bitumen, Polym Degrad Stab (2013) 98(6): 1205-1215. https://doi.org/10.1016/j.polymdegradstab.2013.03.008

[7] M. Nobakht, M.S. Sakhaeifar, Dynamic modulus and phase angle prediction of laboratory aged asphalt mixtures, Constr Build Mater (2018) 190: 740-751. https://doi.org/10.1016/i.conbuildmat.2018.09.160

[8] M. Ling, X. Luo, F. Gu, R.L. Lytton, Time-temperature-aging-depth shift functions for dynamic modulus master curves of asphalt mixtures, Constr Build Mater (2017) 157: 943-951. https://doi.org/10.1016/i.conbuildmat.2017.09.156

[9] S. Xu, A. García, J. Su, Q. Liu, A. Tabaković, E. Schlangen, Self - Healing Asphalt Review: From Idea to Practice, Adv Mater Interf (2018) 1800536. https://doi.org/10.1002/admi.201800536

[10] J. Qiu, Self healing of asphalt mixtures: towards a better understanding of the mechanism, TU Delft, Delft University of Technology, 2012.

[11] A. Tabaković, E. Schlangen, Self-healing technology for asphalt pavements, Self-healing Materials, Springer (2015) 285-306. https://doi.org/10.1007/12 2015 335

[12] Q. Liu, E. Schlangen, Á. García, M. van de Ven, Induction heating of electrically conductive porous asphalt concrete, Constr Build Mater (2010) 24(7): 1207-1213. https://doi.org/10.1016/j.conbuildmat.2009.12.019

[13] Q. Liu, W. Yu, S. Wu, E. Schlangen, P. Pan, A comparative study of the induction healing behaviors of hot and warm mix asphalt, Constr Build Mater (2017) 144: 663-670. https://doi.org/10.1016/j.conbuildmat.2017.03.195

[14] J. Tang, Q. Liu, S. Wu, Q. Ye, Y. Sun, E. Schlangen, Investigation of the optimal self-healing temperatures and healing time of asphalt binders, Constr Build Mater (2016) 113: 1029-1033. https://doi.org/10.1016/j.conbuildmat.2016.03.145

[15] Á. García, E. Schlangen, M. van de Ven, D. van Vliet, Induction heating of mastic containing conductive fibers and fillers, Mater Struct (2011) 44: 499-508. https://doi.org/10.1617/s11527-010-9644-2

[16] Q. Liu, E. Schlangen, M. van de Ven, Induction healing of porous asphalt concrete beams on an elastic foundation, J Mater Civ Eng (2012) 25: 880-885. https://doi.org/10.1061/(ASCE)MT.19435533.0000677

[17] B. Gómez-Meijide, H. Ajam, A. Garcia, S. Vansteenkiste, Effect of bitumen properties in the induction healing capacity of asphalt mixes, Constr Build Mater (2018) 190: 131-139. https://doi.org/10.1016/j.conbuildmat.2018.09.102

[18] Q. Liu, Á. García, E. Schlangen, M. van de Ven, Induction healing of asphalt mastic and porous asphalt concrete, Constr Build Mater (2011) 25: 3746-3752 https://doi.org/10.1016/j.conbuildmat.2011.04.016

[19] B. Gómez-Meijide, H. Ajam, P. Lastra-González, A. Garcia, Effect of ageing and RAP content on the induction healing properties of asphalt mixtures, Constr Build Mater (2018) 179: 468-476. https://doi.org/10.1016/j.conbuildmat.2018.05.121

[20] A. García, M. Bueno, J. Norambuena-Contreras, M.N. Partl, Induction healing of dense asphalt concrete, Constr Build Mater (2013) 49: 1-7. https://doi.org/10.1016/i.conbuildmat.2013.07.105

[21] E. Lizasoain-Arteaga, I. Indacoechea-Vega, P. Pascual-Mu-oz, D. Castro-Fresno, Environmental impact assessment of inductionhealed asphalt mixtures, J Clean Prod (2019) 208: 1546-1556 https://doi.org/10.1016/j.jclepro.2018.10.223

[22] Q. Liu, E. Schlangen, M.F. van de Ven, G. van Bochove, J. van Montfort, Predicting the Performance of the Induction Healing Porous Asphalt Test Section, 7th RILEM International Conference on Cracking in Pavements, Springer (2012) 1081-1089. https://doi.org/10.1007/978-94-007-4566-7 103

[23] D. Sun, G. Sun, X. Zhu, A. Guarin, B. Li, Z. Dai, J. Ling, A comprehensive review on self-healing of asphalt materials: Mechanism, model, characterization and enhancement, Adv Colloid Interfac Sci (2018). https://doi.org/10.1016/j.cis.2018.05.003

[24] L. Zhang, Q. Liu, S. Wu, Y. Rao, Y. Sun, J. Xie, P. Pan, Investigation of the flow and self-healing properties of UV aged asphalt binders, Constr Build Mater (2018) 174: 401-409. https://doi.org/10.1016/i.conbuildmat.2018.04.109 
[25] N. Kringos, R. Khedoe, A. Scarpas, A. de Bondt, On the development of a new test methodology for moisture damage susceptibility of asphalt concrete, 5th International Conference 'Bituminous Mixtures and Pavements', Thessaloniki, Greece (2011) 857 - 867.

[26] A. Tabaković, A. Gibney, C. McNally, M.D. Gilchrist, The influence of recycled asphalt pavement on the fatigue performance of asphalt concrete base courses, ASCE J Mater Civ Eng (2010) 2: 643 - 650. https://doi.org/10.1061/(ASCE)MT.1943-5533.0000093

[27] A. Tabaković, W. Post, D. Cantero, O. Copuroglu, S. Garcia, E. Schlangen, The reinforcement and healing of asphalt mastic mixtures by rejuvenator encapsulation in alginate compartmented fibres, Smart Mater Struct (2016) 25: 084003.

https://doi.org/10.1088/0964-1726/25/8/084003

[28] S. Xu, A. Tabaković, X. Liu, E. Schlangen, Calcium alginate capsules encapsulating rejuvenator as healing system for asphalt mastic, Constr Build Mater (2018) 169: 379-387.

https://doi.org/10.1016/i.conbuildmat.2018.01.046

[29] Z. Wu, L.N. Mohammad, L. Wang, M.A. Mull, Fracture resistance characterization of superpave mixtures using the semi-circular bending test, J ASTM Int (2005) 2: 1-15.

https://doi.org/10.1520/JAI12264

[30] S. Sreedhar, E. Coleri, S.S. Haddadi, Selection of a performance test to assess the cracking resistance of asphalt concrete materials, Constr Build Mater (2018) 179: 285-293.

https://doi.org/10.1016/i.conbuildmat.2018.05.258

[31] E. 12697-44: Bituminous mixtures-Test methods for hot mix asphalt-Part 44: Crack propagation by semi-circular bending test, European Committee for Standardization Brussels, 2010. 